\title{
Relation of ETS transcription factor family member ERG, androgen receptor and topoisomerase $2 \beta$ expression to TMPRSS2-ERG fusion status in prostate cancer
}

\author{
Z. KOLAR ${ }^{1, \star}$, A. BURDOVA ${ }^{1,4}$, T. JAMASPISHVILI' ${ }^{1}$, J. BOUCHAL ${ }^{1}$, R. KUCEROVA ${ }^{2}$, M. BIENOVA ${ }^{2}$, M. KRAL ${ }^{3}$, \\ V. STUDENT 3
}

${ }^{1}$ Department of Clinical and Molecular Pathology, Faculty of Medicine and Dentistry, Palacky University and University Hospital, Olomouc; ${ }^{2}$ Department of Dermatovenereology, Faculty of Medicine and Dentistry, Palacky University and University Hospital, Olomouc; ${ }^{3}$ Department of Urology, Faculty of Medicine and Dentistry, Palacky University and University Hospital, Olomouc; ${ }^{4}$ Laboratore AGEL a.s., Laboratory of Medical Genetics, Novy Jicin, Czech Republic, EU

*Correspondence: kolarz@tunw.upol.cz

Received February 17, 2013 / Accepted April 10, 2013

\begin{abstract}
Fusion of TMPRSS2 with ERG in prostate cells is determined by double-strand DNA breaks induced by androgen signaling and transcription stress. The enzyme topoisomerase $2 \beta$ (TOP2B) mediating DNA processing, plays a important role in DNA cleavage. The aim of this study was to analyse expression of AR, TOP2B and ERG in relation to TMPRSS2-ERG gene rearrangement and relevant clinicopathological characteristics in prostate cancer (CaP). Immunohistochemical staining and FISH were used for investigation. ERG expression in prostate cell lesions positively correlated with levels of TMPRSS2ERG fusion gene $(\mathrm{p}<0.0001)$. The most significant co-expression of ERG was found with AR in CaP ( $\mathrm{p}=0.001)$. Significantly more frequent co-expression of ERG was also revealed with TOP2B $(\mathrm{p}=0.028)$. ERG protein expression did not correlate with CaP differentiation status as we found no significant differences in ERG expression for different Gleason categories. We demonstrated a statistically significant positive correlation between the percentage of cells with fusion gene TMPRSS2ERG in CaP and metastatic potential of tumors $(p=0.011)$. Besides these positive corelations of AR with ERG $(p=0.001)$ and TOP2B with ERG $(\mathrm{p}=0.028)$, we also demonstrated a significant co-expression of AR with TOP2B ( $\mathrm{p}=0.007)$ in CaP. There was a statistically significant increase in the TOP2B H-index in locally advanced $\mathrm{CaP}$ in comparison with localized tumors $(\mathrm{p}=0.046)$. ERG expression correlates with occurrence of TMPRSS2-ERG fusion and with AR-driven malignant transformation. The results indicate that detection of the TMPRSS2-ERG fusion gene and parallel immunohistochemical examination of $\mathrm{AR}, \mathrm{TOP} 2 \mathrm{~B}$ and ERG has diagnostic significance and may be useful in assessing the biological character of the prostate cancer as well as selecting the best treatment.
\end{abstract}

Key words: androgen receptor, ERG, topoisomerase $2 \beta$, TMPRSS2-ERG fusion, prostate cancer

The androgen receptor (AR) is a DNA-binding nuclear transcription factor that regulates gene expression. It is essential for prostate differentiation and maintenance of its differentiation status as well as for the regulation of prostate specific gene expressions such as prostate specific antigen (PSA) and type II transmembrane serine protease (TMPRSS2). In the majority of prostate cancers $(\mathrm{CaP})$ there are fusion genes with oncogenic potential involving $5^{\prime}$-end elements composed of androgen regulated genes and the gene ETS transcription factor family $[1,2]$. This fusion causes aberrant transcription which is andro- gen-driven. Although the mechanisms underlying these gene rearrangements are still unclear, recently it has been shown that fusion of TMPRSS2 with ERG (the Estrogen-Regulated Gene, a member of the ETS transcription factor family) in prostate cells is determined by double-strand DNA breaks induced by androgen signaling in response to transcription stress $[1,3]$. The fusion of both genes is possible owing to near configuration of uncovered DNA areas during androgen receptor-driven chromatin looping [4]. The enzyme topoisomerase $2 \beta$ (TOP2B) mediating DNA processing, plays a major role in 
DNA cleavage. In prostatic intraepithelial neoplasias (PIN), a significantly higher coexpression of AR with TOP2B than in normal prostatic epithelium has been reported $[1,2]$. ERG has specific affinity for the genome with the GGA(A/T) motif which is predominantly present at binding sites for the AR and thus is able to inhibit AR expression and activity. ERG can also induce expression of metalloproteinases and genes involved in the plasminogen activator pathway. For this reason, TMPRSS2ERG fusion plays a crucial role in tumor progression since it affects prostate cell differentiation [5]. Rearrangement breakpoints have been found to be enriched near open chromatin, AR and ERG DNA binding sites in the setting of ETS gene fusion TMPRSS2-ERG. However, in tumors lacking ETS fusion, these relations were found to be reversed. This suggests a link between chromatin or transcriptional regulation and the genesis of genomic aberrations [6]. Some recent studies describe a possible link between the ability of the fusion gene to affect the inflammatory process and functional status of the cyclooxygenase-2 (COX-2, enzyme directing prostaglandin synthesis) mediated pathway. Prostaglandins are able to induce growth of $\mathrm{CaP}$, to regulate expression of enzymes contributing to cancer cell invasiveness, angiogenesis and metastasis [7]. In ERG-positive CaP, high expression of Wnt signal pathway effectors like FZD4 was also detected, which can contribute to cellular phenotype leading to the development of EMT (epithelial-mesenchymal transition) $[7,8]$. There is evidence supporting the hypothesis that the presence of the fusion gene, differentiates two molecular groups within prostate cancer with different behaviour, making the fusion gene a potential therapeutic target [9]. We deduce that parallel examination of TMPRSS2-ERG gene rearrangement and expression of AR, TOP2B and ERG in relation to clinicopathological status can contribute to better tumor specification.

\section{Material and methods}

We analysed the fusion gene TMPRSS2-ERG incidence in relation to expression of AR, TOP2B, ERG and clinicopathological characteristics in $100 \mathrm{CaP}$ patients who had not undergone androgen ablation therapy. Simultaneously, we assessed the same parameters in adjacent foci of prostate intraepithelial neoplasia (PIN) and/or benign prostatic hyperplasia (BPH).

Patients and tissues. The currently analysed cases were randomly selected from the tissue bank of the Department of Clinical and Molecular Pathology, Palacký University and University Hospital, Olomouc. They included archival formalin-fixed, paraffin-embedded tissue samples from 100 patients who had undergone radical prostatectomy (RP) for prostate adenocarcinoma between March 2004 and May 2011. The age, preoperative serum PSA level, pTNM stage, Gleason score, tumor category (localized CaP was defined as T1-T2, N0, M0 while locally advanced $\mathrm{CaP}$ was defined as $\mathrm{T} 3-\mathrm{T} 4, \mathrm{~N} 0-\mathrm{N} 1$, M0) and risk category (low: T1-T2a, Gleason score $\leq 6$ and PSA $\leq 10 \mathrm{ng} \mathrm{ml}^{-1}$, intermediate: at least one of the following situations /T2b-c, Gleason score 7 or PSA higher than 10 and lower than $20 \mathrm{ng} \mathrm{ml}^{-1} /$ and high: at least one of the following situations /T3-T4, Gleason score $>7$ or PSA $>20 \mathrm{ng} \mathrm{ml}^{-1 /}$ ) for all cases were noted from the clinical protocols. Patients did not receive hormonal or radiation therapy before the RP or adjuvant therapy before recurrence. Sample sections were used for FISH analysis and for immunohistochemical detection of AR, TOP2B and ERG. Histopathological diagnosis was confirmed by two independent pathologists on hematoxylin and eosin-stained paraffin sections of each sample before FISH assessment. The morphological criteria for 'normal', 'BPH,' 'PIN' and 'malignant prostatic epithelium' conformed to previously published definitions [10].

Immunohistochemistry. ERG expression was evaluated using a commercial rabbit anti-ERG monoclonal antibody (clone EPR3864; Epitomics, Burlingame, CA, USA) and AR expression using mouse anti-AR monoclonal antobody (clone AR441; Dakocytomation, Glostrup, Denmark). The protocol for the immunohistochemistry was as follows: slides were deparaffinized, exposed to heat-induced antigen retrieval in autoclave for 5 minutes at $121^{\circ} \mathrm{C}$ and $\mathrm{pH} 7.8$ (ERG) or in a microwave oven for 15 minutes in a citrate buffer $\mathrm{pH} 6.0$ (AR) and blocked with preantibody solution (10 min). The antibody against ERG was applied in a dilution 1:400 and AR in a dilution 1:100 both for $60 \mathrm{~min}$ at room temperature. Primary antibodies were visualized using the DAKO enVision Kit (DakoCytomation, Glostrup, Denmark) according to producer recommendations and 3,3'-diaminobenzidine (20 min, Sigma Fast 3,3'-diaminobenzidine tablets, SigmaAldrich, St. Louis, MO). Sections were then counterstained with hematoxylin, dehydrated, cleared, mounted, and covered. Immunohistochemical staining for TOP2B (1:500, polyclonal, Bethyl Laboratories, Inc. Montgomery, TX, USA) was carried out in a BenchMark XT autostainer (Ventana Medical Systems, Tuscon, AZ, USA) using an i-View detection kit (Ventana Medical Systems) and treatment with citrate buffer ( $\mathrm{pH}$ 6.0) in a microwave oven for 15 minutes. Additional procedures were the same as the above. Only nuclear staining of ERG, AR and TOP2B were assessed using an $\mathrm{H}$-score system obtained by multiplying the intensity of the stain ( 0 : no staining; 1 : weak staining; 2 : moderate staining; 3 : intense staining) by the percentage (0 to 100) of cells showing that staining intensity (H-score range, 0 to 300). For negative controls, the primary antibodies were omitted. As positive control for AR, confirmed $\mathrm{AR}$ positive $\mathrm{CaP}$ samples were used. As TOP2B and ERG expression is normally found in lymphocytes and endothelium, these were used as internal positive controls for TOP2B and ERG staining.

TMPRSS2-ERG fusion status analysis by FISH. To confirm the presence of rearrangement at 21q22 where TMPRSS2 and ERG genes are located, we used interphase fluorescence in situ hybridization (FISH). FISH analysis was carried out using Poseidon TMPRSS2-ERG (21q22) Del, Break, TC Probe (Kreatech Diagnostics, Amsterdam, The Netherlands), which is optimised to detect deletion between TMPRSS2 and ERG 

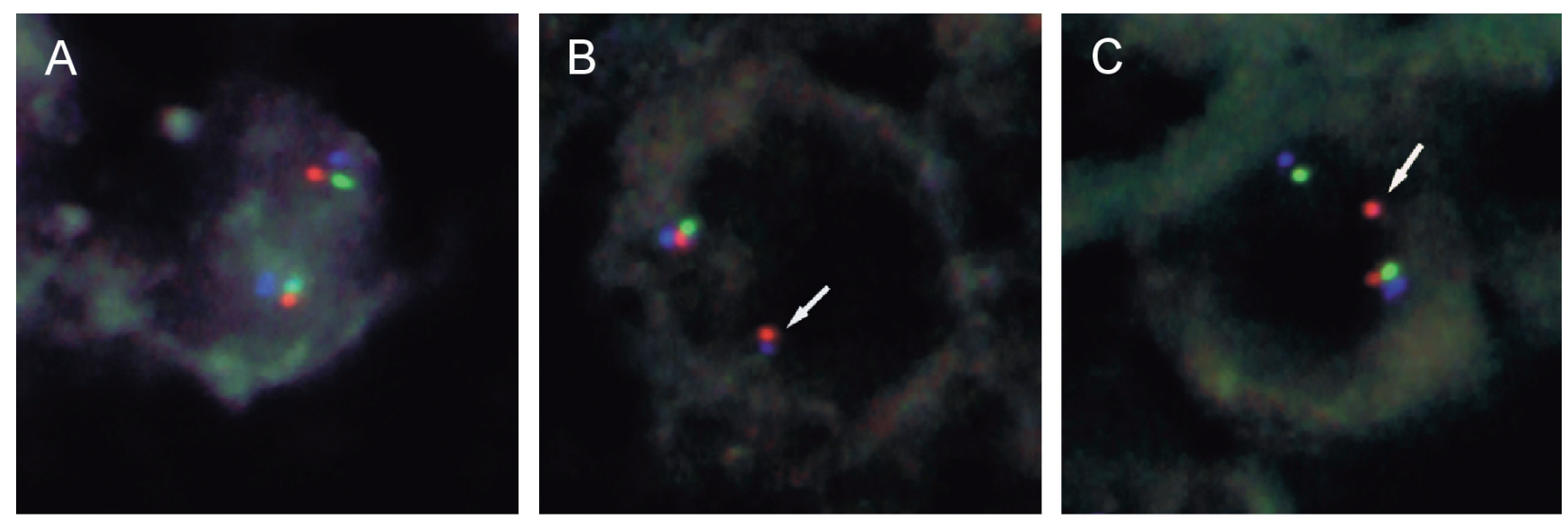

Figure 1. Three-color FISH detection of TMPRSS2-ERG fusion. The signals are: the red one for distal TMPRSS2 gene region, the green one represents intervening sequence between TMPRSS2 and ERG genes and the blue signal represents proximal ERG gene region. format of (A) the nucleus without TMPRSS2-ERG rearrangement demonstrates two clusters of juxtaposed red, green and blue signals. (B) detail of the nucleus with the TMPRSS2-ERG fusion, where the loss of the intervening sequence is observed as a loss of a green signal leaving a red/blue signal at $21 \mathrm{q} 22$ (white arrow). (C) detail of the nucleus with the rearrangement of TMPRSS2 gene which results in a break of the signal cluster, observed as a single red (white arrow) and green/blue signal pattern.

associated with the TMPRSS2-ERG fusion in a triple-color deletion assay. It also detects translocations involving the TMPRSS2 region. Formalin-fixed, paraffin-embedded tissue sections were used for interphase FISH. Deparaffinized tissue was treated with $0.2 \mathrm{M} \mathrm{HCl}$ for 20 minutes, $\mathrm{NaSCN}$ for $30 \mathrm{~min}$ utes at $80^{\circ} \mathrm{C}$ and digested with pepsin (Sigma-Aldrich, USA) for 70 minutes. The tissues and FISH probe were co-denatured for 5 minutes at $80^{\circ} \mathrm{C}$ and hybridized overnight at $37^{\circ} \mathrm{C}$ in a humid chamber (StatSpin ThermoBrite, IRIS, MA). FISH interpretation was carried out by a molecular cytogeneticist and a pathologist, both experienced in analysing interphase FISH experiments. H\&E sections were available for sideby-side comparison with the FISH image to localise tumor cells. Paired benign prostatic epithelium was also evaluated as a negative control. Expected signal patterns according to instructions of the TMPRSS2-ERG probe manual - a nucleus without TMPRSS2-ERG rearrangement demonstrates two pairs of juxtaposed green, red and blue signals. A nucleus with the TMPRSS2-ERG fusion demonstrates loss of green signal leaving a red/blue signal at 21q22, and a nucleus with split of the probe in case a translocation at 21q22 results in a break of the fusion signal. This is observed as a single red and green/blue signal pattern at the derivative chromosomes when only TMPRSS2 is involved. It is observed as a single blue and red/green signal pattern at the derivative chromosomes when only ERG is involved. The samples were analysed under a $100 \mathrm{x}$ oil immersion objective using an Olympus BX-51 fluorescence microscope (Olympus, Center Valley, PA) equipped with appropriate filters, a CCD (charge-coupled device) camera and captured by ISIS software (MetaSystems, Altlussheim, Germany) (Figure 1).

Statistical analysis. The data were analyzed using SPSS 15.0 software package (SPSS Inc., Chicago, IL, USA), and a two- tailed $P$ value of less than 0.05 was considered statistically significant. In the case that the Shapiro-Wilk test of normality revealed non normal distribution, the nonparametric KruskalWallis and Mann-Whitney U-tests with Bonferroni correction were used. The relation of age to other tested parameters was performed by ANOVA. The multiple correlations were assessed using the Spearman coefficient of determination and the categorial data were tested using the $\varkappa^{2}$ test and/or in the case of low numbers, the Fisher exact test.

\section{Results}

ERG expression correlates with occurrence of TMPRSS2ERG fusion. ERG expression in prostate cell lesions positively correlated with amount of TMPRSS2-ERG fusion gene ( $p<0.0001)$. In $100 \%$ of cases with confirmed fusion gene, the $\mathrm{H}$-index was higher than 50 with an average $\mathrm{H}$-index of 178.7. In cases without the fusion gene in only $40 \%$ of cases had the $\mathrm{H}$-index higher than 50 with an average $\mathrm{H}$-index of 70.2 which was significantly lower (Figure 2). On the other hand there were a few $\mathrm{CaP}$ without fusion gene which revealed very high ERG positivity and also $\mathrm{CaP}$ cases with positive gene fusion and low ERG expression compared to PIN areas (Figure 3A-C).

ERG expression correlates with AR-driven malignant transformation. ERG positivity ( $\mathrm{H}$-index $>0$ ) was found in $94 \%$ of the analysed cases of $\mathrm{CaP}$ and in $77 \%$ of tumor adjacent areas of BPH/PIN. However, when the threshold of ERG positivity was adjusted to an $\mathrm{H}$-index $>50$, positivity was shown in $66 \%$ of $\mathrm{CaP}$ but in only $3 \%$ of BPH/PIN. The most significant co-expression of ERG was found with AR in $\mathrm{CaP}(\mathrm{p}=0.001)$. Significantly more frequent co-expression of ERG was revealed also with TOP2B ( $p=0.028)$. We found 


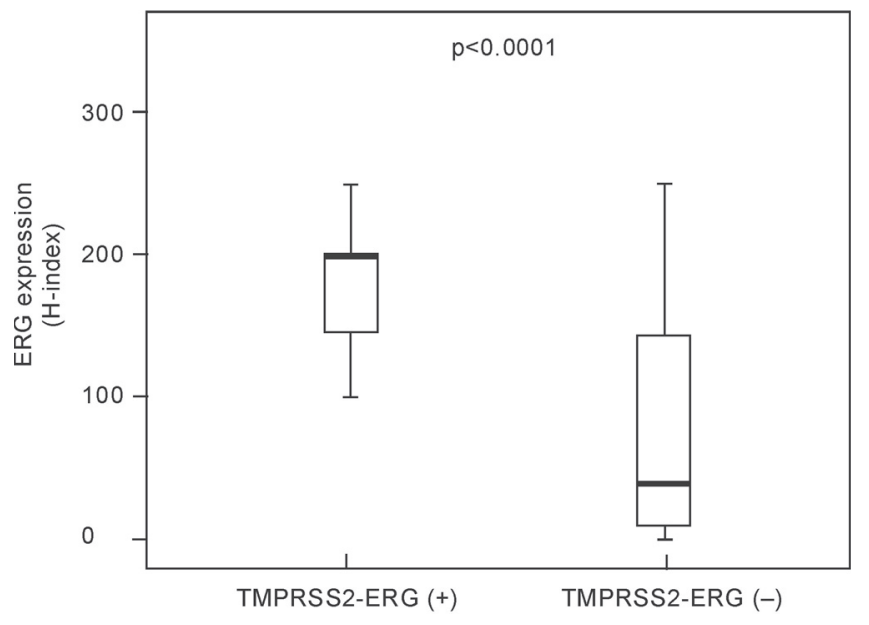

Figure 2. Positive correlation between ERG expression (H-index) and TMPRSS2-ERG fusion gene detection in CaP. Mann-Whitney U-tests with Bonferroni correction $(\mathbf{p}<0.0001)$. no significant co-expression of these molecules in areas of $\mathrm{BPH} / \mathrm{PIN}$ despite the fact that ERG as well as AR and TOP2B positivity was uniformly enhanced in tumor surrounding areas of BPH/PIN (Figure 3D). ERG protein expression did not correlate with $\mathrm{CaP}$ differentiation status as we found no significant differences in ERG expression for different Gleason categories. In cases where we were able to analyse ERG expression in the same tissue section, we observed amplification of ERG expression in PIN compared to $\mathrm{BPH}$ but owing to the small number of samples with joint $\mathrm{BPH}$ and PIN occurrence we were unable to perform any relevant statistical analysis.

TMPRSS2-ERG rearrangement correlates with metastatic potential of CaP. We confirmed positive TMPRSS2-ERG fusion in 20 percents of analysed cases which we compared with cases lacking any TMPRSS2-ERG rearrangement (61\%). The remaining cases (19\%) showed another gene rearrangement which could not be identified by the used probes. We demonstrated a statistically significant positive correlation
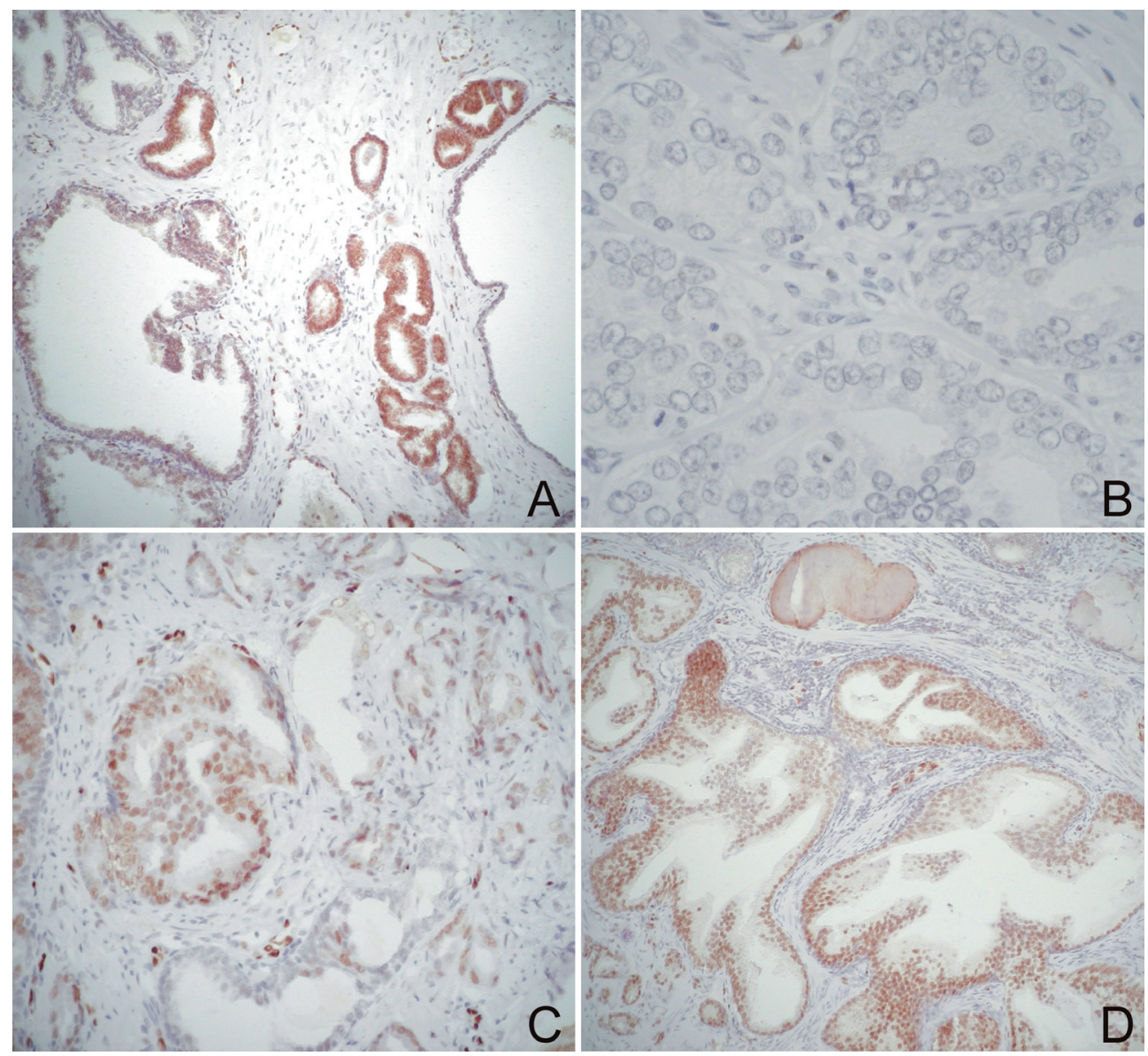

Figure 3. Different expression of ERG in BPH/PIN areas in the vicinity of CaP and in CaP foci: Relation to fusion gene TMPRSS2-ERG status. (A) ERG expression pattern in a sample without fusion gene TMPRSS2-ERG. High expression of ERG in CaP, lower in PIN and ERG negativity in BPH, (B) Another ERG expression pattern in a sample without fusion gene TMPRSS2-ERG. Negativity of ERG expression, (C) High ERG expression in PIN and lower ERG expression in CaP with proven TMPRSS2-ERG fusion gene, (D) Amplified ERG expression on the border between CaP and PIN without relation to fusion gene status. 
between the percentage of cells with gene rearrangement TMPRSS2-ERG in CaP and metastatic potential of tumors. The higher the percent positivity, the more frequent the occurrence of metastasis into lymph nodes $(\mathrm{p}=0.011)$ (Figure 4). The Fisher test confirmed a statistically significant increase in lymph node metastasis in cases of $\mathrm{CaP}$ with $4 \mathrm{n}$ ploidy $(\mathrm{p}=0.049)$. We were unable to find any significant relationships between fusion gene TMPRSS2-ERG, tumor progression (local versus locally advanced) and risk (low, intermediate, high) variables.

ERG, AR and TOP2B co-expression has diagnostic significance. AR positivity was found in all samples of $\mathrm{CaP}$ and levels of its expression positively correlated with ERG $(p=0.001)$. In contrast to generally accepted data, we found no cases of AR negativity in $\mathrm{CaP}$, however, a low $\mathrm{H}$-index $(\leq 50)$ was demonstrated in $14 \%$ of cases without significant differences between groups with positivity or negativity for gene fusion. In evaluating AR positivity in BPH/PIN foci, we had to take into consideration its different distribution. In

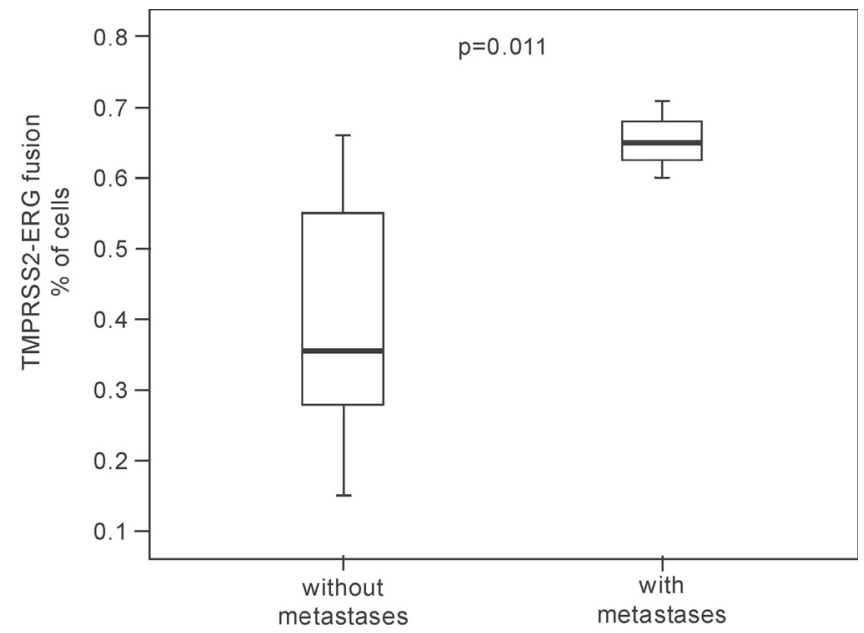

Figure 4. Positive correlation between the percentage of cells with fusion gene TMPRSS2-ERG in CaP and metastatic potential. The higher the percent positivity, the more frequent occurrence of metastasis into lymph nodes $(\mathrm{p}=\mathbf{0 . 0 1 1})$.
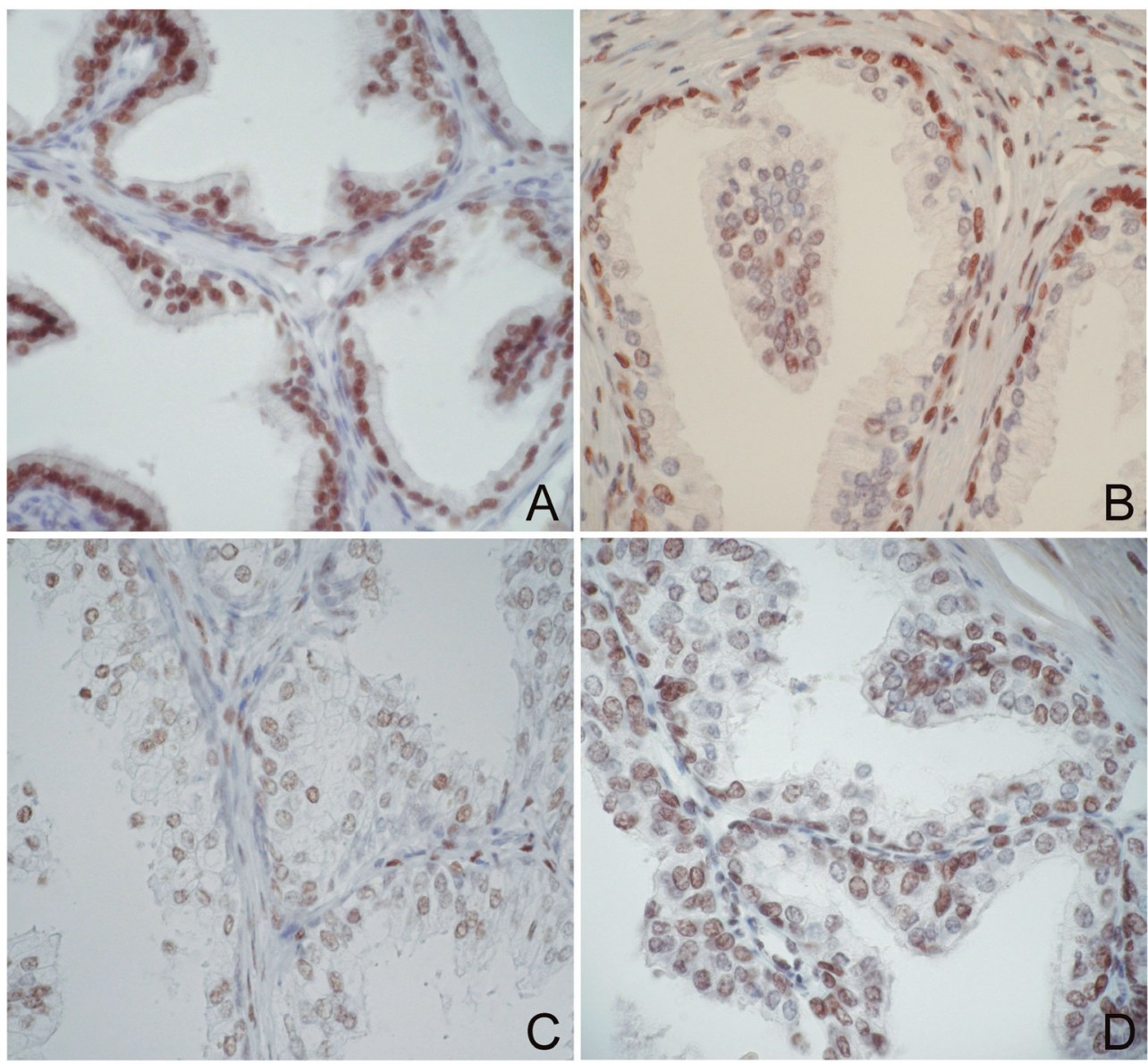

Figure 5. Different expression of AR and TOP2B in BPH/PIN areas in the vicinity of CaP: Relation to fusion gene TMPRSS2-ERG level. (A) Tumor with low fusion gene content, expression of AR in BPH/PIN, (B) The same sample, TOP2B expression in BPH/PIN, (C) Tumor with high fusion gene content, expression of AR in BPH/PIN, (D) The same sample, TOP2B expression in BPH/PIN. High AR activity in BPH/PIN from tumor vicinity with low fusion gene content is accompanied by high TOP2B expression. Low AR activity in BPH/PIN from tumor vicinity with high fusion gene content is accompanied by lower TOP2B expression. 

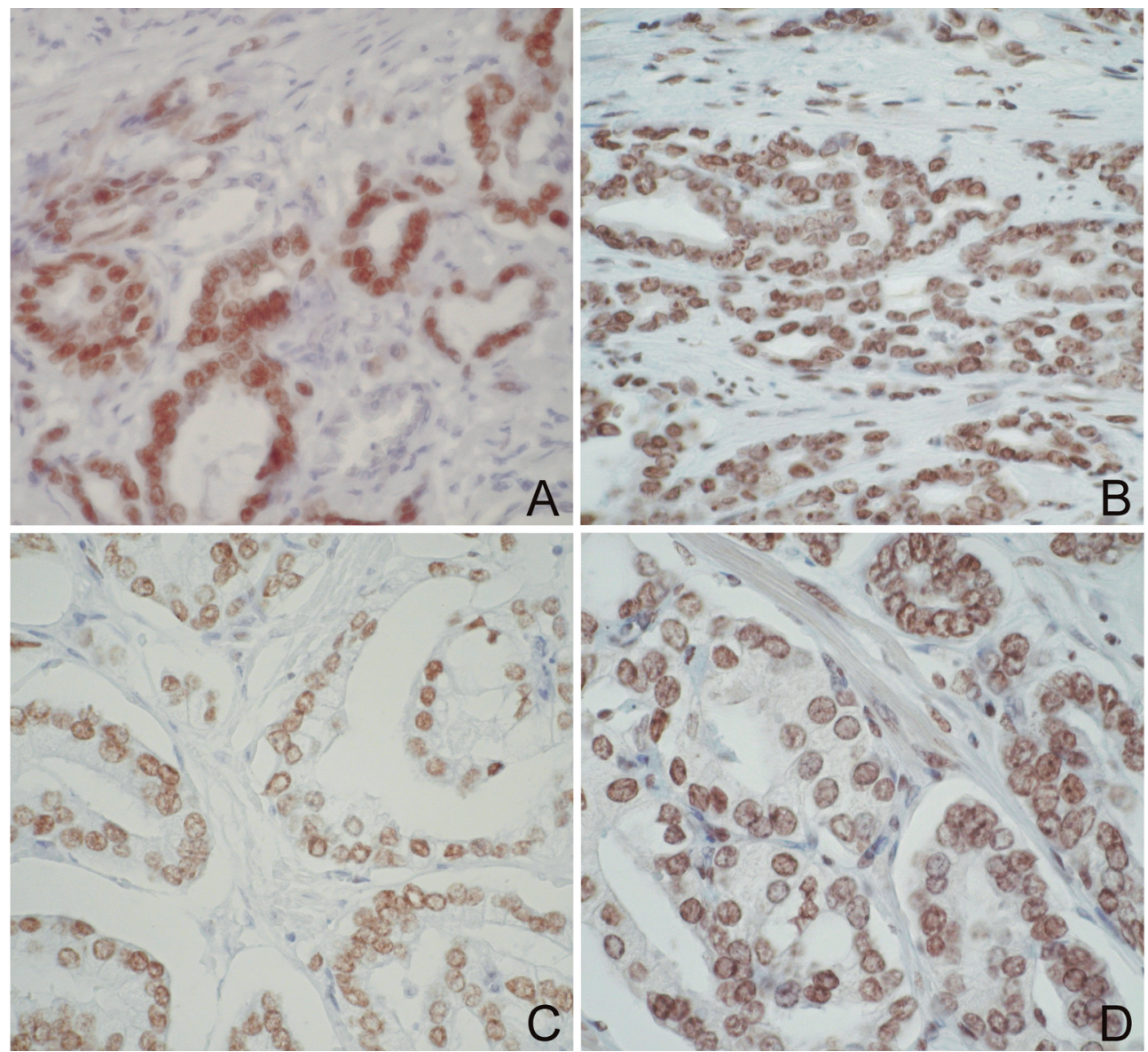

Figure 6. Different expression of AR and TOP2B in CaP foci: Relation to fusion gene TMPRSS2-ERG level. (A) Tumor with low fusion gene content, expression of $\mathrm{AR}$ in $\mathrm{CaP},(\mathrm{B})$ The same sample, TOP2B expression in $\mathrm{CaP},(\mathrm{C})$ Tumor with high fusion gene content, expression of AR in CaP, (D) The same sample, TOP2B expression in CaP. High AR activity in CaP with low fusion gene content is accompanied by high TOP2B expression. Low AR activity in $\mathrm{CaP}$ with high fusion gene content is not accompanied by the corresponding reduction in TOP2B expression.

benign areas, AR positivity prevailed in the luminal cell layer of the prostate gland and only sporadically it was seen in basal myoepithelial layers (Figure 5A). In areas of PIN, we observed AR positivity in all layers of the epithelium (Figure 5C). Like ERG expression, AR expression was also amplified in the parts of the prostate closely surrounding the tumor tissue.

TOP2B expression was also detected in all samples and its levels positively correlated with $\mathrm{AR}(\mathrm{p}=0.007)$ as well as with ERG ( $\mathrm{p}=0.028)$ expression in CaP.

However, there were no significant differences in TOP2B expression between $\mathrm{CaP}$ with positive and negative fusion. The Mann-Whitney U-test confirmed a statistically significant increase in the TOP2B $\mathrm{H}$-index in advanced $\mathrm{CaP}$ in comparison with local tumors $(\mathrm{p}=0.046)$. A useful finding facilitating the diagnosis of border line lesions in the prostate was the different distribution of TOP2B positivity. In $\mathrm{BPH}, \mathrm{TOP} 2 \mathrm{~B}$ was distributed in basal cells of the prostate gland but with continuing malignant transformation (higher PIN grade), the positivity shifted increasingly to luminal cells (Figure 5B,D). A trend was also observed for an association between AR and TOP2B expression in relation to fusion gene level. High AR expression in $\mathrm{BPH} / \mathrm{PIN}$ from tumor vicinity with low fusion gene levels was accompanied by high TOP2B expression. However, low AR expression in $\mathrm{BPH} / \mathrm{PIN}$ from tumor vicinity with high fusion gene content was accompanied by lower TOP2B expression. The situation in $\mathrm{CaP}$ was different. High AR expression in $\mathrm{CaP}$ with low fusion gene content was accompanied by high TOP2B expression while low $\mathrm{AR}$ activity in $\mathrm{CaP}$ with high fusion gene content was not accompanied by a corresponding reduction in TOP2B expression (Figure 6).

\section{Discussion}

The recent literature has described ERG positivity in 50\% $\mathrm{CaP}[5,9,11]$. Our patient group showed ERG positivity in $94 \%$ of $\mathrm{CaP}$ and in $77 \%$ of adjacent $\mathrm{BPH} / \mathrm{PIN}$. However, negativity and/or low positivity of ERG (H-index $\leq 50)$ was found in $34 \%$ of $\mathrm{CaP}$ and $97 \% \mathrm{BPH} / \mathrm{PIN}$. These different results can be 
explained by the different sensitivities of detection method or by setting of the positivity threshold. On the other hand, some authors claim that TMPRSS2-ERG fusion can be induced also in non-malignant prostate epithelial cells [3]. The strongest coexpression of ERG in CaP was with AR ( $\mathrm{p}=0.001)$ and second, there was a significant co-expression of ERG with TOP2B $(\mathrm{p}=0.028)$. We found no comparable relationships in areas of $\mathrm{BPH} / \mathrm{PIN}$ which confirms earlier published findings that ERG occurs in $\mathrm{CaP}$ cells in a 20-100x higher concentration than benign tissues and that it can induce PIN development [7].

We described a significant positive relationship between the percentage of cells with TMPRSS2-ERG fusion gene positivity and $\mathrm{CaP}$ metastasis which is in accord with published data $[12,13]$.

We found no significant difference in ERG expression for different Gleason categories. Our results are not in accordance with two recent studies reporting a negative relation between ERG expression and Gleason score [14,15]. However, it is possible that increasing the number of analysed cases would resolve these differences and refine our results.

ERG protein expression in prostate lesions positively correlated with TMPRSS2-ERG fusion gene content. Overall, 100\% cases with fusion gene positivity showed a higher expression of ERG (H-index $\geq 100)$ while higher levels of ERG were present in only $26 \%$ of negative cases. This finding is in agreement with recently published data $[11,16]$.

AR positivity was found in all cases of CaP. Even here however, to evaluate negativity/positivity, a threshold has to be established and we need to consider the different sensitivities of the immunohistochemical methods used. Given the pathogenesis of TRPMSS2-ERG gene rearrangement it was important to analyse the relationship between AR and TOP2B [2]. Some publications report significantly higher coexpression of both molecules in PIN than in normal prostate epithelium [1]. In our cohort of cases, we found higher co-expression in $\mathrm{CaP}$ than in $\mathrm{BPH} / \mathrm{PIN}$ and we described for the first time topical changes in their distribution in $\mathrm{BPH} / \mathrm{PIN}$ in comparison to normal prostatic epithelium. TOP2B expression did not change in tumors with positive gene rearrangement.

Increased ERG positivity in the vicinity of malignant lesions may be significant. The mechanisms of ERG induction in normal and premalignant cells may be mediated via gene rearrangement dependent as well as independent pathways. Given the simultaneous higher expression of AR and TOP2B, it is probably an androgen-driven process. It should, however, be taken into account that although there is a causal relation between ERG and AR expression there are also AR-negative $\mathrm{CaPs}$ with ERG expression. Minner and co-workers confirmed in $\mathrm{CaP}$ a 95\% concordance between ERG detected by immunohistochemistry and ERG gene rearrangement detected by FISH [11]. ERG expression however was unrelated to clinical outcome and tumor phenotype. These authors also found that AR expression was significantly higher in ERG positive $\mathrm{CaP}$. Our results are in good agreement with these findings.
Thus, the differences in AR expression in ERG-positive and ERG-negative cases could explain the different response of $\mathrm{CaP}$ patients to hormonal ablation.

Detection of the ERG protein and the TMPRSS2-ERG fusion gene, appears to be very promising diagnostically and predictively, particularly in connection with newly developed antibody which is able to bind specifically to ERG fused with TMPRSS2 epitope $[17,18]$.

Overall, our results indicate that detection of the TMPRSS2ERG fusion gene as well as parallel immunohistochemical examination of the three proteins involved in androgen-drived signal pathways may be useful in assessing the biological character of the prostate cancer and selecting the best treatment.

Acknowledgements: This work was supported in part by grants IGA MZ CR NS 9940 and NT11201.

\section{References}

[1] HAFFNER MC, ARYEE MJ, TOUBAJI A, ESOPI DM, ALBADINE $\mathrm{R}$ et al. Androgen-induced TOP2B-mediated double- strand breaks and prostate cancer gene rearrangements. Nat Genet 2010; 42: 668-675. http://dx.doi. org/10.1038/ng.613

[2] BARTEK J, HAMERLIK P, LUKAS J. On the origin of prostate fusion oncogenes. Nat Genet 2010; 42: 647-648. http://dx.doi. org/10.1038/ng0810-647

[3] BASTUS NC, BOYD LK, MAO X, STANKIEWICZ E, KUDAHETTI SC et al. Androgen-induced TMPRSS2: ERG fusion in nonmalignant prostate epithelial cells. Cancer Res 2010; 70: 9544-9548. http://dx.doi.org/10.1158/0008-5472. CAN-10-1638

[4] WU D, ZHANG C, SHEN Y,NEPHEW K, WANG Q. Androgen receptor-driven chromatin Looping in prostate cancer. Trends Endocrinol Metab 2011; 22: 474-480. http://dx.doi. org/10.1016/j.tem.2011.07.006

[5] YU J, YU J, MANI RS, CAO Q, BRENNER CJ et al. An integrated network of androgen receptor, polycomb, and TMPRSS2-ERG gene fusions in prostate cancer progression. Cancer Cell 2010; 17: 443-454. http://dx.doi.org/10.1016/ j.ccr.2010.03.018

[6] BERGER MF, LAWRENCE MS, DEMICHELIS F, DRIER Y, CIBULSKIS $\mathrm{K}$ et al. The genomic complexity of primary human prostate cancer. Nature 2011; 470: 214-220. http://dx.doi. org/10.1038/nature09744

[7] MARTIN SK, KYPRIANOU N. Gene fusions find an ERG-way to tumor inflammation. Cancer Biol Ther 2011; 11: 418-420. http://dx.doi.org/10.4161/cbt.11.4.14499

[8] MOHAMED AA, TAN SH, SUN C, SHAHEDUZZAMAN S, HU Y. et al. ERG oncogene modulates prostaglandin signaling in prostate cancer cells. Cancer Biol Ther 2011; 11: 410-417. http://dx.doi.org/10.4161/cbt.11.4.14180

[9] FERNANDEZ-SERRA A, RUBIO-BRIONES J, GARCIACASADO Z, SOLSONA E, LOPEZ- GUERRERO JA. Cancer de próstata: la revolucion de los genes de fusion. [Prostate cancer: the revolution of the fusion genes]. 
Actas Urol Esp 2011; 35: 420-428. http://dx.doi.org/10.1016/ j.acuroe.2010.11.001

[10] BOSTWICK DG, CHENG L., editors. Urologic Surgical Pathlology. Mosby Elsevier Inc., 2008.

[11] MINNER S, ENODIEN M, SIRMA H, LUEBKE AM, KROHN A et al. ERG status is unrelated to PSA recurrence in radically operated prostate cancer in the absence of antihormonal therapy. Clin Cancer Res 2011; 17: 5878-5888. http://dx.doi. org/10.1158/1078-0432.CCR-11-1251

[12] PERNER S, DEMICHELIS F, BEROUKHIM R, SCHMIDT FH, MOSQUERA JM et al. TMPRSS2: ERG fusion-associated deletions provide insight into the heterogeneity of prostate cancer. Cancer Res 2006; 66: 8337-8341. http://dx.doi. org/10.1158/0008-5472.CAN-06-1482

[13] DEMICHELIS F, FALL K, PERNERS, ANDREN O, SCHMIDT $F$ et al. TMPRSS2: ERG gene fusion associated with lethal prostate cancer in a watchful waiting cohort. Oncogene 2007; 26: 4596-4599. http://dx.doi.org/10.1038/sj.onc.1210237

[14] BISMAR TA, DOLPH M, TENG LH, LIU S, DONNELLY B. ERG protein expression reflects hormonal treatment response and is associated with Gleason score and prostate cancer spe- cific mortality. Eur J Cancer 2012; 48: 538-46. http://dx.doi. org/10.1016/j.ejca.2012.01.001

[15] KRON K, LJU L, TRUDEL D, KRON K, LJU L, TRUDEL D. Correlation of ERG expression and DNA methylation biomarkers with adverse clinicopathologic features of prostate cancer. Clin Cancer Res 2012; 18: 2896-2904. http://dx.doi. org/10.1158/1078-0432.CCR-11-2901

[16] CHAUX A, ALBADINE R, TOUBAJI A, HICKS J, MEEKER A et al. Immunohistochemistry for ERG expression as a surrogate for TMPRSS2-ERG fusion detection in prostatic adenocarcinomas. Am J Surg Pathol 2011; 35: 1014-1020. http://dx.doi.org/10.1097/PAS.0b013e31821e8761

[17] PARK K, TOMLINS SA, MUDALIAR KM, CHIU YL, ESGUEVA R et al. Antibody-based detection of ERG rearrangement-positive prostate cancer. Neoplasia 2010; 12: 590-598.

[18] VAN LEENDERS GJ, BOORMANS JL, VISSERS CJ, HOOGLAND AM, BRESSERS AA et al. Antibody EPR3864 is specific for ERG genomic fusions in prostate cancer: implications for pathological practice. Mod Pathol 2011; 24: 1128-1138. http://dx.doi.org/10.1038/modpathol.2011.65 